\title{
Filaggrin genotype associations with atopic march in children
}

\author{
Volodymyr O. Dytiatkovskyi, Olexandr E. Abaturov \\ SI “Dnipropetrovsk Medical Academy of the Ministry of Health of Ukraine”, Ukraine
}

ABSTRACT

Introduction: Atopic diseases affect children of all age groups, composing the atopic march (AM) - the progression of atopic inflammation from the skin to upper and lower airways, finally leading to bronchial asthma, with not enough elucidated genetic associations.

Aim: We performed the first study in Ukraine of the single nucleotide polymorphism (SNP) rs7927894 of filaggrin (FLG) gene associations with atopic dermatitis (AD), seasonal allergic rhino-conjunctivitis, perennial allergic rhinitis, and bronchial asthma at children.

Material and methods: Genotyping of FLG gene variants by real-time polymerase chain reaction-restriction fragment length polymorphism (PCR-RFLP) in 119 atopic patients of the main group and 34 non-atopic patients of the control group aged 2-17 years, and IgE testing, were performed.

Results: Performed correlation analysis of rs7927894 FLG variants detected significant prevalence of the $\mathrm{C} / \mathrm{T}$ variant in the atopic patients compared to non-atopic ( $\mathrm{OR}=2.52 ; p<0.05)$. T/T genotype of FLG is significantly 5 -fold more strongly associated with the male gender than female $(\mathrm{OR}=5.95 ; p<0.05)$. The dataset obtained shows a strong association of C/T genotype of rs7927894 FLG with AD and BA incidence $(\mathrm{OR}=3.01$ and $\mathrm{OR}=4.47 ; p<0.05)$.

Conclusions: Male atopic patients carrying the SNP rs7927894 T/T variant of the FLG gene have a 5-fold stronger significant association with atopic diseases than non-carriers of such genotype. For the first time in Ukraine there has been detected a significant association between the C/T rs7927894 genotype variant of the FLG gene in pediatric patients suffering from atopic dermatitis and bronchial asthma. A possible association ( $p=0.066$ ) between the SNP rs7927894 C/T of the FLG gene and perennial allergic rhinitis requires studies on larger patient cohorts.

\section{KEY WORDS:}

filaggrin, single nucleotide polymorphism, children, atopic march, bronchial asthma.

\section{ADDRESS FOR CORRESPONDENCE:}

Volodymyr Olexandrovych Dytiatkovskyi, SI "Dnipropetrovsk Medical Academy of the Ministry of Health of Ukraine”, Ukraine, e-mail: ditiatkovskyvo@gmail.com 


\section{INTRODUCTION}

The atopic march (AM) manifests as progression of different allergic atopic diseases frequently observed in children with genetically predisposed hyper-IgE-antibody responses to common environmental allergens [1]. Atopic march in its natural history is often initiated by atopic dermatitis (AD), as allergic atopic inflammation in skin is now considered as the major gateway for the onset of AM [2]. Thus, progression of AM manifests with the consecutive involvement of mucosae - seasonal allergic rhino-conjunctivitis (SARC), perennial allergic rhinitis (PAR) and bronchial asthma (BA). During the last decade, the paradigm of AM was revised to reveal the associations of its manifestations with genotype variations - single nucleotide polymorphisms (SNPs) [3]. Still today, the role of SNPs is incompletely understood as part of the mechanism of atopic diseases' pathogenesis in children. A breakthrough in understanding of AM mechanisms was the discovery of the filaggrin $(F L G)$ loss-of-function mutations that had provided evidence of skin barrier defects' associations with consequent development of $\mathrm{AD}$ and BA [4]. Epidemiological studies witness the concept of AM initiated by AD and followed by BA $[5,6]$.

Particularly, SNP rs7927894 of the FLG gene is reported to have an association with occurrence of $A D$ and persistent PAR [7]. Recent studies report that FLG defects are also associated with $\mathrm{AD}$-independent risk of development of SARC, PAR and BA - i.e. AD-negative AM [8]. Consequently, a large genome-wide association study showed that genetic variations of rs7927894 in chromosome region $11 \mathrm{q} 13.5$ were highly associated in approximately $13 \%$ of homozygous individuals of European origin with risk of developing AD 1.47-fold higher compared to non-carriers [9]. This hypothesis has been supported by multiple studies on populations with Caucasian ancestry as the number of $\mathrm{AD}$ risk loci reported in individuals of European ancestry equals 11, which equates to $14.4 \%$ for the heritability of $\mathrm{AD}[10,11]$.

\section{AIM}

For the first time in Ukraine to study the associations of the single nucleotide polymorphism (SNP) rs7927894 of the FLG gene with major AM nosologies in children atopic dermatitis, seasonal allergic rhino-conjunctivitis, perennial allergic rhinitis, bronchial asthma.

\section{MATERIAL AND METHODS}

In our study, we recruited 119 ill children aged from 2 to 17 years (mean age: $7.9 \pm 0.3$ years) into the main group with AM nosologies: AD, SARC, PAR, BA. The age-gender distribution in the main group was as follows: males -76 (63.9\%), females $-43(36.1 \%), p<0.001$. The incidence of $\mathrm{AM}$ diagnosis among patients of the main group was as follows: $\mathrm{AD}=52(43.7 \%), \mathrm{SARC}=57(47.9 \%), \mathrm{PAR}=48$ $(40.3 \%), \mathrm{BA}=19(16.0 \%)$. The number of combined comorbidity cases among the main group was 48 (40.3\%) patients. In all children of the main group the atopic nature of diseases was confirmed by increased levels of total IgE and specific $\operatorname{IgE}$ in serum to the major components of case-specific allergens performed on the ImmunoCap machine using the microarrays for immune fluorescence reaction.

The control group consisted of 34 non-atopic children aged from 3 to 17 years old with digestive tract pathologies who had neither any allergy clinical manifestations nor any individual or family allergy history. The age-gender distribution was as follows: male - 16 (47.1\%), female - 18 (52.9\%), mean age: $11.0 \pm 0.7$ years. These patients were suffering from gastrointestinal tract pathologies, e.g.: functional dyspepsia, chronic gastritis, chronic gastroduodenitis, peptic ulcer, bile stones.

All the patients were genotyped using the regular rs7927894, allelic discrimination TaqMan assays for polymerase chain reaction-restriction fragment length polymorphism (PCR-RFLP). Genotyping was performed on the Applied Biosystems 7500 Fast Real Time PCR System at the Laboratory of the Department for General and Molecular Pathophysiology of Kyiv Bohomolets National Institute of Physiology. The material for RT-PCR was obtained by a buccal patch using one-use sterile scrubs, then stored at $-32^{\circ} \mathrm{C}$ in the freezer. The PCR was done with a volume of 10-15 $\mu$ l using the default thermal profile. The target DNA was extracted from samples using the NeoPrep ${ }^{100}$ set. We considered only SNP with minor allele frequency $>5 \%$ and considered the results with a $p$-value below 0.05 significant.

\section{STATISTICAL ANALYSIS}

The statistical analysis was performed using Statistica v.6.1 (StatSoft Inc., USA, license \# AGAR909E415822FA) software set with application of Spearman rank-order correlation $(\mathrm{R})$ for measuring the strength and direction of association between two ranked variables, Pearson $\chi^{2}$ test and Fisher exact two-tailed test (FET) for the comparison of relative values, logistic regression for calculating the odds ratio (OR) of the atopic diseases occurrence with a 95\% confidence interval (CI) - R, FET and Pearson chisquare being validated by the $p$-value level.

\section{RESULTS}

We detected no statistically significant difference between the gender composition in the main and control 
groups (Table 1), which shows that these groups are gender comparable (FET $p=0.111$ ).

We detected a statistically significant difference in the age composition of the groups (Table 2) which shows that atopic diseases vary from the non-atopic ones in their onset and peak incidence age.

Correlation analysis of rs7927894 variants within the main and control groups demonstrated significant prevalence of $\mathrm{C} / \mathrm{T}$ variant in the atopic patients compared to non-atopic subjects (Table 3 ).

The other correlation dataset gives an insight into the gender associations of rs7927894 - the T/T variant is significantly 5 -fold more strongly associated with male than female gender (Table 4).

We also applied the abovementioned analysis tools to the specific nosologies of AM.

TABLE 1. Gender composition of the main and control groups

\begin{tabular}{|l|c|c|}
\hline $\begin{array}{l}\text { Gender/statistical } \\
\text { values }\end{array}$ & Main group & Control group \\
\hline Male & $63.9 \%$ & $47.1 \%$ \\
\hline Female & $36.1 \%$ & $52.9 \%$ \\
\hline FET, two-tailed, $p$-value & \multicolumn{2}{|c|}{0.111} \\
\hline
\end{tabular}

\section{ATOPIC DERMATITIS}

Atopic dermatitis was detected in 52 (43.7\%) patients in the main group. Mean age in this cohort was 7.54 years old (95\% CI: 6.6-8.4): minimum 2 years and maximum 14 years. In the gender composition there were more male patients $(57.7 \%)$ than female ones $(42.3 \%)$. Figure 1 shows the prevalence of moderate severity (71.2\%) AD compared to mild (17.3\%) and severe (11.5\%) forms.

We obtained results supporting the hypothesis $[1,6,7]$ of rs7927894 having the strongest association with AD (Table 5).

We found a significant 3-fold OR for suffering AD within carriers of the C/T variant of the SNP studied. Also, the C/T genotype was significantly the most fre-

TABLE 2. Age composition of the main and control groups

\begin{tabular}{|l|c|c|}
\hline Age [years] & Main group & Control group \\
\hline $0-3$ & $5.0 \%$ & $2.9 \%$ \\
\hline $4-6$ & $34.5 \%$ & $11.8 \%$ \\
\hline $7-11$ & $47.1 \%$ & $38.2 \%$ \\
\hline $12-18$ & $13.4 \%$ & $47.1 \%$ \\
\hline Pearson $\chi^{2}, p$-value & \multicolumn{2}{|c|}{$<0.001$} \\
\hline
\end{tabular}

TABLE 3. Genotype structure for rs7927894 FLG in the main and control groups

\begin{tabular}{|l|c|c|c|}
\hline \multirow{2}{*}{ Groups and values } & \multicolumn{2}{|c|}{ SNP rs7927894 genotype variants $F L G, \%$} \\
\cline { 2 - 4 } & $\mathrm{C} / \mathrm{C}$ & $\mathrm{C} / \mathrm{T}$ & 16.0 \\
\hline Main group $(n=119)$ & 40.3 & 43.7 & 20.6 \\
\hline Control group $(n=34)$ & 55.9 & 23.5 & 0.605 \\
\hline FET two-tailed, $p$-value & 0.120 & 0.046 & $-0.051(0.530)$ \\
\hline Spearman, $r(p$-value) & $-0.130(0.108)$ & $+0.172(0.034)$ & 0.73 \\
\hline OR & 0.53 & 2.52 & $0.28-1.94$ \\
\hline $95 \% \mathrm{Cl}$ & $0.25-1.16$ & $1.05-6.07$ & \\
\hline
\end{tabular}

TABLE 4. Gender associations of rs7927894 FLG in the main group of atopic patients

\begin{tabular}{|l|c|c|c|}
\hline \multirow{2}{*}{$\begin{array}{l}\text { Gender (male/female) } \\
\text { and values }\end{array}$} & C/C & C/T & T/T \\
\cline { 2 - 4 } Male patients $(n=76)$ & 35.5 & 42.1 & 22.4 \\
\hline Female patients $(n=43)$ & 48.8 & 46.5 & 4.7 \\
\hline FET two-tailed, $p$-value & 0.177 & 0.702 & 0.017 \\
\hline Spearman, $r(p$-value) & $+0.130(0.158)$ & $+0.043(0.645)$ & $-0.232(0.011)$ \\
\hline OR & 0.58 & 0.84 & 5.91 \\
\hline $95 \% \mathrm{Cl}$ & $0.27-1.24$ & $0.39-1.79$ & $1.27-27.40$ \\
\hline
\end{tabular}




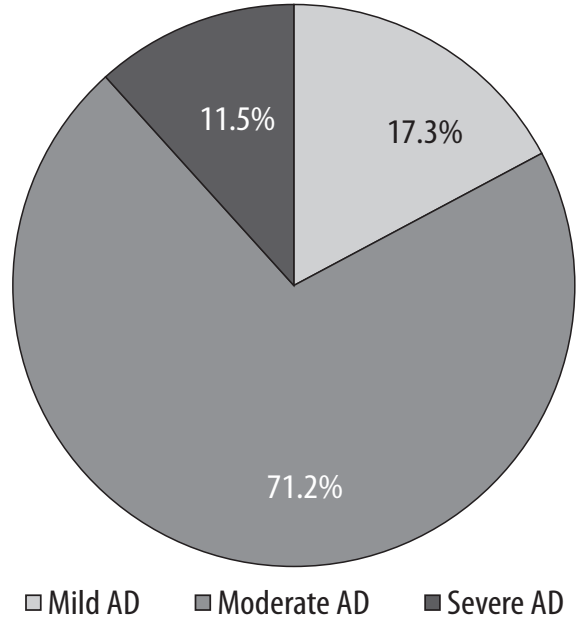

FIGURE 1. Severity structure of atopic dermatitis (AD) in patients of the main group

quent variant within the AD cohort of rs7927894 in the main group.

\section{SEASONAL ALLERGIC RHINO-CONJUNCTIVITIS}

Seasonal allergic rhino-conjunctivitis (SARC) was detected in 57 (47.9\%) patients of the main group. Mean age in this cohort was 8.19 years old (95\% CI: 7.3-9.1): minimum 3 years and maximum 17 years. In the gender composition there were more male patients $(71.9 \%)$ than female ones $(28.1 \%)$. All the cases of SARC in our study were persistent ones. Figure 2 shows the severity structure of the SARC cohort given that there were no cases of severe SARC in this study.

We found no significant associations $(p>0.05)$ of SARC with any variant of rs7927894 (Table 6).

Still, we detected that the $\mathrm{C} / \mathrm{C}$ and $\mathrm{C} / \mathrm{T}$ genotypes had the highest frequency among all the rs7927894 genotypes in patients with SARC $-45.6 \%$ and $33.3 \%$ respectively.

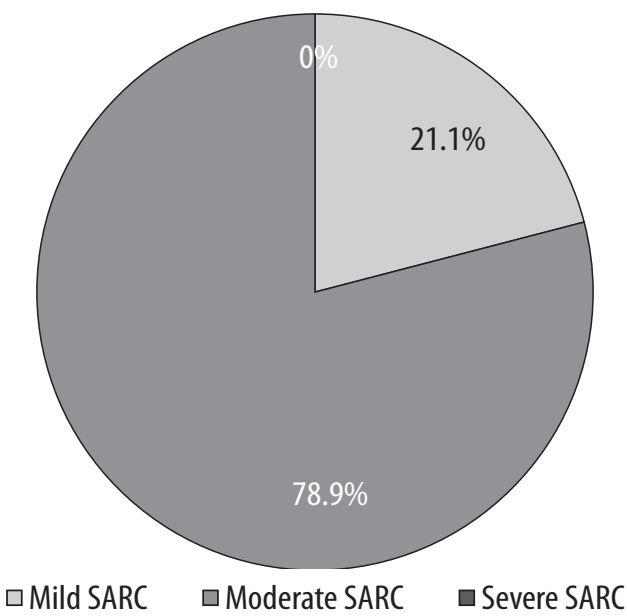

FIGURE 2. Severity structure of seasonal allergic rhino-conjunctivitis (SARC) in patients of the main group
TABLE 5. Genotype structure of rs7927894 FLG in patients with AD compared to control group

\begin{tabular}{|l|c|c|c|}
\hline \multirow{2}{*}{ Group/values } & \multicolumn{3}{|c|}{ rs7927894 FLG } \\
\cline { 2 - 4 } & $\mathrm{C} / \mathrm{C}$ & $\mathrm{C} / \mathrm{T}$ & $\mathrm{T} / \mathrm{T}$ \\
\hline Main group & $38.5 \%$ & $48.1 \%$ & $13.5 \%$ \\
\hline Control group & $55.9 \%$ & $23.5 \%$ & $20.6 \%$ \\
\hline FET two-tailed, $p$-value & 0.127 & 0.025 & 0.390 \\
\hline OR & 0.49 & 3.01 & 0.60 \\
\hline 95\% Cl & $0.20-1.20$ & $1.13-7.98$ & $0.19-1.93$ \\
\hline
\end{tabular}

\section{PERENNIAL ALLERGIC RHINITIS}

Perennial allergic rhinitis was detected in 48 (40.3\%) patients of the main group. Mean age in this cohort was 8.42 years old (95\% CI: 7.4-9.4): minimum 3 years and maximum 17. In the gender composition there were more male patients $(66.7 \%)$ than female ones $(33.3 \%)$. There was detected predominance of moderate persistent PAR, while intermittent form was detected in one-third of patients, and no severe PAR was reported (Figure 3).

Although not statistically significant, there was more than 2-fold higher likelihood of developing PAR among carriers of C/T rs7927894 variant in the main group $(\mathrm{OR}=2.53 ; p=0.066$; Table 7$)$.

\section{BRONCHIAL ASTHMA}

Bronchial asthma (BA) was detected in 19 (16.0\%) patients of the main group. Mean age in this cohort was 7.53 years old (95\% CI: 6.0-9.1): minimum 4 years old and maximum 15 years. In the gender composition there was a slight excess of male patients (57.9\%) over female ones $(42.1 \%)$, although the numbers were comparable. The severity and controllability structure of BA are given respectively in Figures 4 and 5.

The genotype allelic discrimination dataset shows a strong $(\mathrm{OR}=4.47 ; 95 \% \mathrm{CI}: 1.30-15.39, p<0.05)$ as-

TABLE 6. Genotype structure of rs7927894 FLG in patients with SARC compared to control group

\begin{tabular}{|l|c|c|c|}
\hline \multirow{2}{*}{ Groups/values } & \multicolumn{3}{|c|}{ rs7927894 FLG } \\
\cline { 2 - 4 } & $\mathrm{C} / \mathrm{C}$ & $\mathrm{C} / \mathrm{T}$ & $\mathrm{T} / \mathrm{T}$ \\
\hline Main group & $45.6 \%$ & $33.3 \%$ & $21.1 \%$ \\
\hline Control group & $55.9 \%$ & $23.5 \%$ & $20.6 \%$ \\
\hline FET two-tailed, $p$-value & 0.391 & 0.354 & 1.000 \\
\hline 0R & 0.66 & 1.63 & 1.03 \\
\hline $95 \% \mathrm{Cl}$ & $0.28-1.57$ & $0.61-4.32$ & $0.36-2.97$ \\
\hline
\end{tabular}




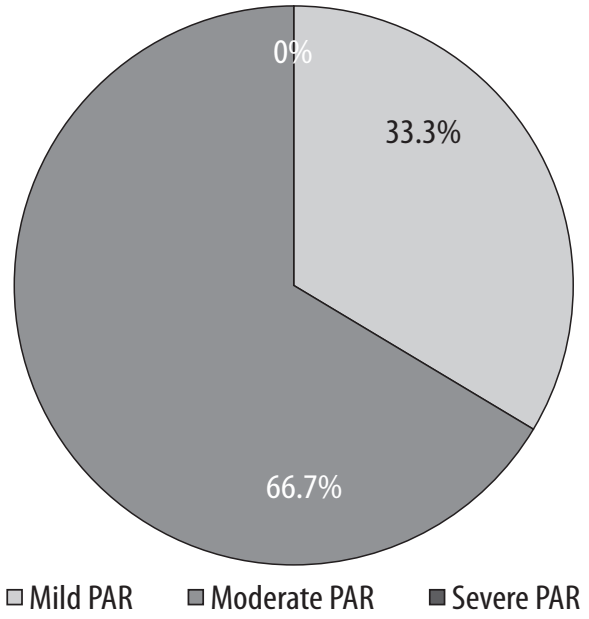

FIGURE 3. Severity structure of perennial allergic rhinitis (PAR) in patients of the main group

sociation of C/T rs7927894 genotype with BA incidence (Table 8).

\section{DISCUSSION}

We detected the highest incidence of the C/T rs7927894 variant among the atopic patients of the main group with a significant, more than 2 -fold association $(\mathrm{OR}=2.52)$ with occurrence of diseases composing the AM. Still, we also detected a significant and strong association of T/T rs7927894 variant and patients' gender - males have a 5-fold higher risk of developing atopic diseases than females $(\mathrm{OR}=5.91, p<0.05)$.

Our other findings comply with recent population-based studies which support the hypothesis of AM in the general population $[5,6]$. Thus, we detected a statistically significant association between rs7927894 C/T genotype variant and $\mathrm{AD}$ - prevalence of $48.1 \%$ and a 3.01

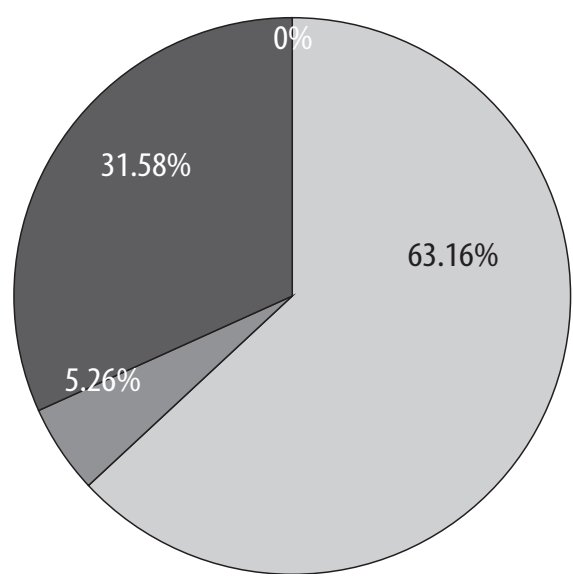

$\square$ Intermittent $\quad \square$ Mild persistent

$\square$ Moderate persistent $\quad$ Severe persistent

FIGURE 4. The bronchial asthma severity degree structure in patients of the main group
TABLE 7. Genotype structure of rs7927894 FLG in patients with PAR compared to control group

\begin{tabular}{|l|c|c|c|}
\hline \multirow{2}{*}{ Groups/values } & \multicolumn{3}{|c|}{ rs7927894 FLG } \\
\cline { 2 - 4 } & $\mathrm{C} / \mathrm{C}$ & $\mathrm{C} / \mathrm{T}$ & $\mathrm{T} / \mathrm{T}$ \\
\hline Main group & $41.7 \%$ & $43.8 \%$ & $14.6 \%$ \\
\hline Control group & $55.9 \%$ & $23.5 \%$ & $20.6 \%$ \\
\hline FET two-tailed, $p$-value & 0.263 & 0.066 & 0.557 \\
\hline OR & 0.56 & 2.53 & 0.66 \\
\hline $95 \% \mathrm{Cl}$ & $0.23-1.39$ & $0.94-6.81$ & $0.20-2.13$ \\
\hline
\end{tabular}

OR $(p<0.05)$ - evidence for the predisposition within carriers of this genotype for AD. Also, we detected the strongest association between rs7927894 C/T genotype variant and BA - prevalence of $57.9 \%$ and a $4.47 \mathrm{OR}$ $(p<0.05)$ - strong evidence of an association between the variant mentioned above and risk of $\mathrm{AD}$ transformation into BA within the AM course. We also observed a possible $\mathrm{C} / \mathrm{T}$ variant association with $\mathrm{PAR}(\mathrm{OR}=2.53$,

TABLE 8. Genotype structure of rs7927894 FLG in patients with BA compared to control group

\begin{tabular}{|l|c|c|c|}
\hline \multirow{2}{*}{ Groups/values } & \multicolumn{3}{|c|}{ rs7927894 FLG } \\
\cline { 2 - 4 } & $\mathrm{C} / \mathrm{C}$ & $\mathrm{C} / \mathrm{T}$ & $\mathrm{T} / \mathrm{T}$ \\
\hline Main group & $31.6 \%$ & $57.9 \%$ & $10.5 \%$ \\
\hline Control group & $55.9 \%$ & $23.5 \%$ & $20.6 \%$ \\
\hline FET two-tailed, $p$-value & 0.150 & 0.018 & 0.463 \\
\hline OR & 0.36 & 4.47 & 0.45 \\
\hline 95\% Cl & $0.11-1.22$ & $1.30-15.39$ & $0.08-2.55$ \\
\hline
\end{tabular}

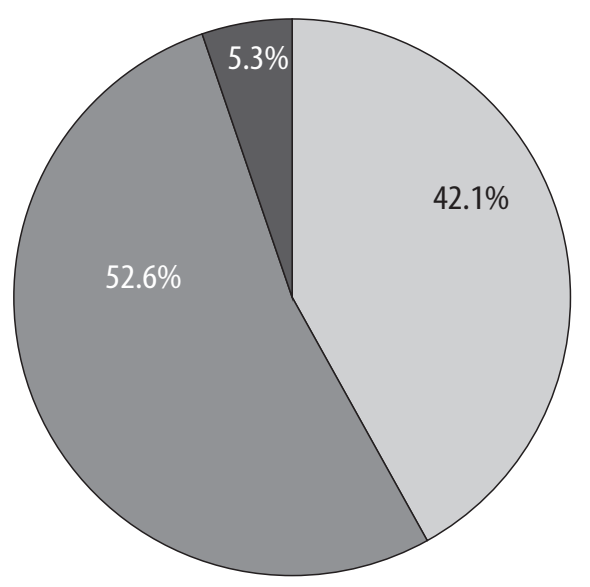

$\square$ Well-controlled $\quad \square$ Partly controlled $\quad$ Uncontrolled FIGURE 5. The bronchial asthma control structure in patients of the main group 
$p=0.066)$ which necessitates studies on larger patient cohorts.

\section{CONCLUSIONS}

C/T genotype variant of rs7927894 of the FLG gene is associated with the development of AM diseases and is the most frequent among nosologies - AD, PAR, BA. T/T genotype variant rs7927894 of the FLG gene is a risk factor for AM progression in male pediatric patients. C/T genotype variant rs7927894 of the FLG gene has a significantly strong association with AD and BA which confirms the association of pathogenesis for these two diseases. C/T genotype variant rs7927894 of the FLG gene has a possible association with PAR which requires studies on larger patient cohorts.

\section{CONFLICT OF INTEREST}

The authors declare no conflict of interest.

\section{REFERENCES}

1. Marenholz I, Esparza-Gordillo J, Rüschendorf F, et al. Meta-analysis identifies seven susceptibility loci involved in the atopic march. Nat Commun 2015; 6: 8804 .

2. Han H, Roan F, Ziegler SF. The atopic march: current insights into skin barrier dysfunction and epithelial cell-derived cytokines. Immunol Rev 2017; 278: 116-30.

3. Li M. Current evidence of epidermal barrier dysfunction and thymic stromal lymphopoietin in the atopic march. Eur Respir Rev 2014; 23: 292-8.

4. Palmer CN, Irvine AD, Terron-Kwiatkowski A, et al. Common lossof-function variants of the epidermal barrier protein filaggrin are a major predisposing factor for atopic dermatitis. Nat Genet 2006; 38: 441-6.

5. Saunes M, Øien T, Dotterud CK, et al. Early eczema and the risk of childhood asthma: a prospective, population-based study. BMC Pediatr 2012; 12: 168.

6. von Kobyletzki LB, Bornehag CG, Hasselgren M, et al. Eczema in early childhood is strongly associated with the development of asthma and rhinitis in a prospective cohort. BMC Dermatol 2012; 12: 11 .

7. Ponińska JK, Samoliński B, Tomaszewska A, et al. Haplotype dependent association of rs7927894 (11q13.5) with atopic dermatitis and chronic allergic rhinitis: a study in ECAP cohort. PLoS One 2017; 12: e0183922.

8. van den Oord RA, Sheikh A. Filaggrin gene defects and risk of developing allergic sensitisation and allergic disorders: systematic review and meta-analysis. Br Med J 2009; 339: b2433.

9. Esparza-Gordillo J, Weidinger S, Fölster-Holst R, et al. A common variant on chromosome 11q13 is associated with atopic dermatitis. Nat Genet 2009; 41: 596-601.

10. O'Regan GM, Campbell LE, Cordell HJ, et al. Chromosome 11q13.5 variant associated with childhood eczema: an effect supplementary to filaggrin mutations. J Allergy Clin Immunol 2010; 125: 170-4. e1-2.
11. Ellinghaus D, Baurecht H, Esparza-Gordillo J, et al. High-density genotyping study identifies four new susceptibility loci for atopic dermatitis. Nat Genet 2013; 45: 808-12. 